\title{
EXPERIMENT OF UNIQUE COMBINATION NUMBER DUE TO THE THIRD-ORDER NEUTRON-CORRELATION
}

\author{
Tomohiro Endo $^{1}$, Sho Imai ${ }^{1}$, Kenichi Watanabe ${ }^{1}$, Akio Yamamoto ${ }^{1}$ \\ Atsushi Sakon ${ }^{2}$, Kengo Hashimoto ${ }^{2}$, Masao Yamanaka ${ }^{3}$, and Cheol Ho Pyeon ${ }^{3}$ \\ ${ }^{1}$ Nagoya University \\ Furo-cho, Chikusa-ku, Nagoya-shi, 464-8603, Japan \\ ${ }^{2}$ Kindai University \\ 3-4-1, Kowakae, Higashiosaka-shi, Osaka, 557-8502, Japan \\ ${ }^{3}$ Kyoto University \\ Asashiro-nishi, Kumatori-cho, Sennan-gun, Osaka, 590-0494, Japan \\ t-endo@energy.nagoya-u.ac.jp, imai.sho@a.mbox.nagoya-u.ac.jp, \\ k-watanabe@energy.nagoya-u.ac.jp, a-yamamoto@energy.nagoya-u.ac.jp, \\ sakon@kindai.ac.jp, kengoh@pp.iij4u.or.jp, \\ m-yamanaka@rri.kyoto-u.ac.jp, pyeon@rri.kyoto-u.ac.jp
}

\begin{abstract}
From zero-power reactor noise measurement, the second- and third-order neutron correlation factors $Y$ and $\mathcal{Y}_{3}$ can be evaluated by analyzing mean, variance, the third-order central moment of neutron count data. Theoretically, it is expected that the neutron-correlation ratio $y_{3} / Y^{2}$ converges to the unique combination number " 3 " at a near-critical state in an arbitrary system without depending on the fissile material and the neutron-energy spectrum of core, as the neutron counting gate width $T$ increases sufficiently. Thus, the information about the difference between $y_{3} / Y^{2}$ and " 3 " has the potential to judge whether a target unknown system is critical or not and to roughly guess the absolute value of subcriticality. In this study, the detector dead-time effect on $\mathcal{Y}_{3} / Y^{2}$ is theoretically investigated based on the heuristic method using the single-, pair-, and trio-detection probabilities with the fundamental mode approximation. As a result, it is clarified that the saturation value of $y_{3} / Y^{2}$ converges to " 3 " independent of the dead time, when a target system is a critical state. For validation, actual experimental results are presented for a non-multiplication system driven by ${ }^{252} \mathrm{Cf}$ spontaneous source, and shallow and deep subcritical systems at Japanese experimental facilities (UTRKINKI and KUCA) under the shutdown state. Consequently, it is demonstrated that $\mathcal{Y}_{3} / Y^{2}$ shows a significant difference from " 3 " in the non-multiplication system. In the case of subcritical systems driven by inherent neutron sources, it is confirmed that the ratios $\mathcal{Y}_{3} / Y^{2}$ are close to the unique combination number " 3 ," and the slight difference from " 3 " is measurable by the long-time reactor noise measurement for the deep subcritical system.
\end{abstract}

KEYWORDS: reactor noise, subcriticality, third-order neutron correlation, dead-time 


\section{INTRODUCTION}

In this study, the theoretical formula for the third-order neutron-correlation technique is derived to take the dead-time effect in a neutron-detection process into account. In addition, experimental results of the thirdorder neutron-correlation technique are presented for a non-multiplication system driven ${ }^{252} \mathrm{Cf}$ source and subcritical systems at the Japanese reactor-physics-experimental facilities, the Kindai University Training and Research Reactor (UTR-KINKI) and the Kyoto University Critical Assembly (KUCA).

We have been investigating the subcriticality measurement technique for an unknown system, where the detailed information about nuclide composition and geometry is not available for the preliminary numerical analysis to evaluate some required parameters, such as the point kinetics parameters (e.g. neutron generation time $\Lambda$ and effective delayed neutron fraction $\beta_{\text {eff }}$ ) and a spatial correlation factor. In the previous study [1], it was theoretically clarified that the neutron-correlation ratio of the third-order factor $\mathcal{Y}_{3}$ to the squared value of the second-order factor $Y$ converges to the unique combination number " 3 " at a near-critical state in an arbitrary system, as the neutron counting gate width increases sufficiently. This unique combination number corresponds to the double factorial $(2 n-3)$ !! when $n=3$, which is the combination number of binary tree in a fission-chain-family having two two-forked branches. As discussed in the previous study [1], by carefully analyzing the difference between the neutron-correlation ratio $y_{3} / Y^{2}$ and " 3 ," the statistical test for the evaluation of the critical state and the absolute measurement of subcriticality can be achieved.

In the proposed technique, there are still the following issues to be addressed. One of the issues is the detector dead time effect on the ratio $\mathcal{Y}_{3} / Y^{2}$, which might occurs under a situation where the neutron count rate is high compared with the dead-time of the neutron counter. Another issue is an actual experimental validation for a non-multiplication system and a deeper subcritical system.

Thus, in this study, we aimed to newly derive the theoretical formulae for $\mathcal{Y}_{3}$ and $\mathcal{Y}_{3} / Y^{2}$ based on a heuristic method to treat the dead-time effect. The theoretical derivation is explained in Section 2. Furthermore, as presented in Section 3, we also aimed to obtain actual experimental results for a nonmultiplication system driven by a ${ }^{252} \mathrm{Cf}$ spontaneous fission source, using an inhouse neutron detector to achieve the high detection efficiency. For comparison, actual experimental results for two subcritical systems carried out at UTR-KINKI and KUCA were also presented in Section 4.

\section{THIRD-ORDER NEUTRON CORRELATION TECHNIQUE}

In this section, the theory for the third-order neutron correlation technique was briefly explained. As compared with previous studies [1,2], we aimed to derive the theoretical formulae by taking the dead-time effect in a neutron-detection process into account.

\subsection{Single-, Pair-, and Trio-detection Probabilities}

Let us assume that that neutron counts $C$ are detected within a counting gate width $T$ for a steady state of a source-driven subcritical system. Then, the second- and third-order neutron correlation factors $Y$ and $\mathcal{Y}_{3}$ are evaluated as follows:

$$
Y \equiv \frac{\left\langle(C-\langle C\rangle)^{2}\right\rangle}{\langle C\rangle}-1=\frac{\langle C(C-1)\rangle-\langle C\rangle^{2}}{\langle C\rangle}
$$




$$
\begin{array}{r}
\mathscr{y}_{3} \equiv\left(\frac{\left\langle(C-\langle C\rangle)^{3}\right\rangle}{\langle C\rangle}-1\right)-3\left(\frac{\left\langle(C-\langle C\rangle)^{2}\right\rangle}{\langle C\rangle}-1\right) \\
=\frac{\langle C(C-1)(C-2)\rangle-3\langle C(C-1)\rangle\langle C\rangle+2\langle C\rangle^{3}}{\langle C\rangle},
\end{array}
$$

where the brackets \langle\rangle indicate the expected value. The correlation factors $Y$ and $\mathcal{Y}_{3}$ represent measures of the relative deviations in variance and skewness from those of the Poisson distribution.

Based on a heuristic method with the fundamental mode approximation [1-3], the following single-, pair-, and trio-detection probabilities $\left(P_{1}\left(t_{1}\right) d t_{1}, P_{2}\left(t_{1}, t_{2}\right) d t_{1} d t_{2}\right.$, and $\left.P_{3}\left(t_{1}, t_{2}, t_{3}\right) d t_{1} d t_{2} d t_{3}\right)$ are firstly derived to obtain the theoretical formulae for Eqs. (1) and (2):

$$
\begin{gathered}
P_{1}\left(t_{1}\right) d t_{1}=R d t_{1}, \\
P_{2}\left(t_{1}, t_{2}\right) d t_{1} d t_{2} \approx\left(R^{2}+\frac{R}{2}\left(\frac{\mathcal{F}_{2}}{-\rho \mathcal{F}_{1}}+\frac{\mathcal{S}_{2}}{\mathcal{S}_{1}}\right) \mathcal{D} \mathrm{e}^{-\alpha\left(t_{2}-t_{1}\right)}\right) d t_{1} d t_{2}, \\
P_{3}\left(t_{1}, t_{2}, t_{3}\right) d t_{1} d t_{2} d t_{3} \\
\approx\left(R^{3}+\frac{R^{2}}{2}\left(\frac{\mathcal{F}_{2}}{-\rho \mathcal{F}_{1}}+\frac{\mathcal{S}_{2}}{\mathcal{S}_{1}}\right) \mathcal{D}\left(\mathrm{e}^{-\alpha\left(t_{3}-t_{2}\right)}+\mathrm{e}^{-\alpha\left(t_{3}-t_{1}\right)}+\mathrm{e}^{-\alpha\left(t_{2}-t_{1}\right)}\right)\right. \\
+\frac{R}{2}\left(\frac{\mathcal{F}_{2}}{-\rho \mathcal{F}_{1}}+\frac{\mathcal{S}_{2}}{\mathcal{S}_{1}}\right) \frac{\mathcal{F}_{2}}{-\rho \mathcal{F}_{1}} \mathcal{D}^{2} \mathrm{e}^{-\alpha\left(t_{2}-t_{1}\right)-\alpha\left(t_{3}-t_{2}\right)} \\
\left.+\frac{R}{3}\left(\frac{\mathcal{F}_{3}}{-\rho \mathcal{F}_{1}}+\frac{\mathcal{S}_{3}}{\mathcal{S}_{1}}\right) \mathcal{D}^{2} \mathrm{e}^{-\alpha\left(t_{2}-t_{1}\right)-\alpha\left(t_{3}-t_{1}\right)}\right) d t_{1} d t_{2} d t_{3},
\end{gathered}
$$

where $-\rho$ is subcriticality; $\alpha$ is prompt neutron decay constant; $R$ is neutron count rate; and other notations are defined as follows using forward and adjoint eigenfunctions $\psi_{0}(\vec{r}, E, \vec{\Omega})$ and $\psi_{0}^{\dagger}(\vec{r}, E, \vec{\Omega})$ :

$$
\begin{gathered}
\mathcal{D} \equiv \int_{V} d V \int_{0}^{\infty} d E \Sigma_{\mathrm{d}}(\vec{r}, E) \phi_{0}(\vec{r}, E), \\
\mathcal{F}_{n} \equiv \int_{V} d V \int_{0}^{\infty} d E \Sigma_{\mathrm{f}}(\vec{r}, E) \phi_{0}(\vec{r}, E) \sum_{v=0}^{\infty} \frac{v !}{(v-n) !} p_{\mathrm{f}}(v, \vec{r}, E)\left(\bar{\psi}_{0, \mathrm{f}}^{\dagger}(\vec{r})\right)^{n}, \\
\mathcal{S}_{n} \equiv \int_{V} d V S(\vec{r}) \sum_{q=0}^{\infty} \frac{q !}{(q-n) !} p_{\mathrm{s}}(q, \vec{r})\left(\bar{\psi}_{0, \mathrm{~s}}^{\dagger}(\vec{r})\right)^{n}, \\
\phi_{0}(\vec{r}, E) \equiv \int_{4 \pi} \psi_{0}\left(\vec{r}, E, \vec{\Omega}^{\prime}\right) d \Omega^{\prime}, \\
\bar{\psi}_{0, \mathrm{f}}^{\dagger}(\vec{r}) \equiv \int_{0}^{\infty} d E^{\prime} \int_{4 \pi} d \Omega^{\prime} \frac{\chi_{\mathrm{f}}\left(\vec{r}, E^{\prime}\right)}{4 \pi} \psi_{0}^{\dagger}\left(\vec{r}, E^{\prime}, \vec{\Omega}^{\prime}\right), \\
\bar{\psi}_{0, \mathrm{~s}}^{\dagger}(\vec{r}) \equiv \int_{0}^{\infty} d E^{\prime} \int_{4 \pi} d \Omega^{\prime} \frac{\chi_{\mathrm{s}}\left(\vec{r}, E^{\prime}\right)}{4 \pi} \psi_{0}^{\dagger}\left(\vec{r}, E^{\prime}, \vec{\Omega}^{\prime}\right),
\end{gathered}
$$

where $\chi_{\mathrm{f}}$ and $\chi_{\mathrm{s}}$ are energy spectra of fission and external neutron source, respectively; $p_{\mathrm{f}}$ and $p_{\mathrm{s}}$ are probability distributions of $v$ - and $q$-neutrons per fission and source-decay, respectively; $S(\vec{r})$ is the spatial distribution of external neutron source; other symbols are conventional meanings in the reactor physics.

These single-, pair-, and trio-detection probabilities represent the probabilities for detecting one, a pair, and a trio of neutrons at each time $\left(t_{1}, t_{2}, t_{3}\right)$, respectively. The pair-detection probability $P_{2}\left(t_{1}, t_{2}\right) d t_{1} d t_{2}$ is also known as the Rossi- $\alpha$ histogram [3,4]. The probability densities of $P_{2}\left(t_{1}, t_{2}\right)$ and $P_{3}\left(t_{1}, t_{2}, t_{3}\right)$ increase because of the branching process in fission-chain due to the fission and source-decay. 


\subsection{Dead-time Effect on Neutron Correlation Factors}

Using detection probabilities shown in Eqs. (3)-(5), the expected numbers of single-neutron, pairs and trios detected during the counting gate width $T$ can be derived to obtain the theoretical formulae for the neutron correlation factors $Y$ and $\mathcal{Y}_{3}$. Based on the heuristic idea proposed by the previous study [5], the dead-time effect in the neutron-detection process can be taken into account in these expected numbers of neutrons when $T>2 \tau$ :

$$
\begin{gathered}
\langle C\rangle=\int_{0}^{T} d t_{1} P_{1}\left(t_{1}\right), \\
\frac{\langle C(C-1)\rangle}{2}=\int_{0}^{T-\tau} d t_{1} \int_{t_{1}+\tau}^{T} d t_{2} P_{2}\left(t_{1}, t_{2}\right), \\
\frac{\langle C(C-1)(C-2)\rangle}{6}=\int_{0}^{T-2 \tau} d t_{1} \int_{t_{1}+\tau}^{T-\tau} d t_{2} \int_{t_{2}+\tau}^{T} d t_{3} P_{3}\left(t_{1}, t_{2}, t_{3}\right),
\end{gathered}
$$

where $\tau$ is dead-time of neutron-detector. Note that the detection efficiency $\mathcal{D}$ in Eqs. (12)-(14) is corrected to consider the dead-time effect, e.g., by multiplying the original $\mathcal{D}$ by a probability of counting-loss due to the dead-time.

By applying Eqs. (12)-(14) to Eqs. (1) and (2) with Eqs. (3)-(5), the theoretical formulae for the neutron correlation neutron factors $Y(T, \tau)$ and $\mathcal{Y}_{3}(T, \tau)$ can be finally obtained as follows:

$$
\begin{aligned}
& Y(T, \tau) \approx\left(\frac{\mathcal{F}_{2}}{-\rho \mathcal{F}_{1}}+\frac{\mathcal{S}_{2}}{\mathcal{S}_{1}}\right) \frac{\mathcal{D}}{\alpha}\left(\left(1-\frac{\tau}{T}\right) \mathrm{e}^{-\alpha \tau}-\frac{\mathrm{e}^{-\alpha \tau}-\mathrm{e}^{-\alpha T}}{\alpha T}\right)-R \tau\left(2-\frac{\tau}{T}\right) \\
& \mathcal{Y}_{3}(T, \tau) \approx 3\left(\frac{\mathcal{F}_{2}}{-\rho \mathcal{F}_{1}}+\frac{\mathcal{S}_{2}}{\mathcal{S}_{1}}\right) \frac{\mathcal{F}_{2}}{-\rho \mathcal{F}_{1}}\left(\frac{\mathcal{D}}{\alpha}\right)^{2}\left(\left(1-2 \frac{\tau}{T}\right)\left(\mathrm{e}^{-2 \alpha \tau}+\mathrm{e}^{-\alpha T}\right)-2 \frac{\mathrm{e}^{-2 \alpha \tau}-\mathrm{e}^{-\alpha T}}{\alpha T}\right) \\
&+\left(\frac{\mathcal{F}_{3}}{-\rho \mathcal{F}_{1}}+\frac{\mathcal{S}_{3}}{\mathcal{S}_{1}}\right)\left(\frac{\mathcal{D}}{\alpha}\right)^{2}\left(\left(1-2 \frac{\tau}{T}\right) \mathrm{e}^{-3 \alpha \tau}-\frac{3 \mathrm{e}^{-3 \alpha \tau}-4 \mathrm{e}^{-\alpha(T+\tau)}+\mathrm{e}^{-\alpha(2 T-\tau)}}{2 \alpha T}\right) \\
&+R^{2} \tau^{2}\left(9-8 \frac{\tau}{T}\right) \\
&-3 R \tau\left(\frac{\mathcal{F}_{2}}{-\rho \mathcal{F}_{1}}+\frac{\mathcal{S}_{2}}{\mathcal{S}_{1}}\right) \frac{\mathcal{D}}{\alpha}\left\{\frac{(1+3 \alpha \tau) \mathrm{e}^{-\alpha \tau}-\mathrm{e}^{-2 \alpha \tau}}{\alpha \tau}\right. \\
&\left.-2 \frac{\left(1-\left(\mathrm{e}^{-\alpha \tau}-2 \alpha \tau\right)(\alpha \tau+1)\right) \mathrm{e}^{-\alpha \tau}-\left(\mathrm{e}^{\alpha \tau}-1+\alpha \tau\right) \mathrm{e}^{-\alpha T}}{\alpha \tau \alpha T}\right\}
\end{aligned}
$$

In order to clarify the dead-time effect on the third-order neutron correlation technique, the saturation values of $Y_{\infty}$ and $\mathcal{Y}_{3, \infty}$ with respect to sufficiently large $T$ can be derived as follows:

$$
\begin{gathered}
Y_{\infty}(\tau)=\lim _{T \rightarrow \infty} Y(T, \tau) \approx\left(\frac{\mathcal{F}_{2}}{-\rho \mathcal{F}_{1}}+\frac{\mathcal{S}_{2}}{\mathcal{S}_{1}}\right) \frac{\mathcal{D}}{\alpha} \mathrm{e}^{-\alpha \tau}-2 R \tau \\
\mathcal{Y}_{3, \infty}(\tau)=\lim _{T \rightarrow \infty} Y_{3, \infty}(T, \tau) \\
\approx 3\left(\frac{\mathcal{F}_{2}}{-\rho \mathcal{F}_{1}}+\frac{\mathcal{S}_{2}}{\mathcal{S}_{1}}\right) \frac{\mathcal{F}_{2}}{-\rho \mathcal{F}_{1}}\left(\frac{\mathcal{D}}{\alpha}\right)^{2} \mathrm{e}^{-2 \alpha \tau}+\left(\frac{\mathcal{F}_{3}}{-\rho \mathcal{F}_{1}}+\frac{\mathcal{S}_{3}}{\mathcal{S}_{1}}\right)\left(\frac{\mathcal{D}}{\alpha}\right)^{2} \mathrm{e}^{-3 \alpha \tau}+9 R^{2} \tau^{2} \\
-3 R \tau\left(\frac{\mathcal{F}_{2}}{-\rho \mathcal{F}_{1}}+\frac{\mathcal{S}_{2}}{\mathcal{S}_{1}}\right) \frac{\mathcal{D}}{\alpha}\left(\frac{(1+3 \alpha \tau) \mathrm{e}^{-\alpha \tau}-\mathrm{e}^{-2 \alpha \tau}}{\alpha \tau}\right) .
\end{gathered}
$$


As can be seen from Eq. (17), the dead-time effect reduces the magnitude of the second-order neutron correlation $Y_{\infty}(\tau)$ by an exponential term $\mathrm{e}^{-\alpha \tau}$ and a linear function $-2 R \tau$. On the other hand, in the case of the third-order neutron correlation factor $\mathcal{Y}_{3, \infty}(\tau)$, there are not only reduction terms but also a quadratic gain term $+9 R^{2} \tau^{2}$ owing to the dead-time effect.

As the subcritical system becomes closer to the criticality state where the fundamental-mode approximation is reasonably applicable, the ratio $\mathcal{Y}_{3, \infty} / Y_{\infty}^{2}$ can be well approximated by the following linear function with respect to the subcriticality $(-\rho)$ :

$$
\frac{\mathcal{Y}_{3, \infty}}{Y_{\infty}^{2}}=\lim _{\substack{T \rightarrow \infty \\-\rho \ll 1}} \frac{\mathcal{Y}_{3, \infty}(T, \tau)}{(Y(T, \tau))^{2}} \approx 3+(-\rho) \frac{\mathcal{F}_{1}}{\mathcal{F}_{2}}\left(\frac{\mathcal{F}_{3}}{\mathcal{F}_{2}} \mathrm{e}^{-\alpha \tau}-3 \frac{\mathcal{S}_{2}}{\mathcal{S}_{1}}+3 R \tau \frac{\alpha}{\mathcal{D}} \mathrm{e}^{\alpha \tau}\left(1-\frac{1-\mathrm{e}^{-\alpha \tau}}{\alpha \tau}\right)\right) .
$$

It is interestingly noted that, just in the critical state, $\mathcal{Y}_{3, \infty} / Y_{\infty}^{2}$ converges to unique combination number " 3 ," independent of the fissile material $\left(\mathcal{F}_{n}\right)$, prompt neutron decay constant $\alpha$, detection efficiency $\mathcal{D}$, and dead time $\tau$. In an unknown subcritical system, the magnitude of difference between $\mathcal{Y}_{3, \infty} / Y_{\infty}^{2}$ and " 3 " roughly corresponds to the order of subcriticality $-\rho[1,2]$. Thus, the information about $\left|\mathcal{Y}_{3, \infty} / Y_{\infty}^{2}-3\right|$ is useful to judge whether the system is critical or not and to estimate the absolute value of $-\rho$.

If a target system is a non-multiplication system driven by a multiple-emission source such as ${ }^{252} \mathrm{Cf}$ spontaneous fission source, the ratio $\mathcal{Y}_{3, \infty} / Y_{\infty}^{2}$ converges to a specific number depending on the multiplicity of the external source $\left(\delta_{n}\right)$, dead-time $\tau$, prompt neutron decay constant $\alpha$, and count rate $R$ :

$$
\frac{y_{3, \infty}}{Y_{\infty}^{2}} \approx \frac{\mathcal{S}_{1} \mathcal{S}_{3} \mathrm{e}^{-3 \alpha \tau}+3 R \tau \mathcal{S}_{1} \frac{\alpha}{\mathcal{D}}\left(3 R \tau \mathcal{S}_{1} \frac{\alpha}{\mathcal{D}}-\mathcal{S}_{2}\left(\frac{(1+3 \alpha \tau) \mathrm{e}^{-\alpha \tau}-\mathrm{e}^{-2 \alpha \tau}}{\alpha \tau}\right)\right)}{\left(\mathcal{S}_{2} \mathrm{e}^{-\alpha \tau}-2 R \tau \mathcal{S}_{1} \frac{\alpha}{\mathcal{D}}\right)^{2}} .
$$

For simplicity, if $\tau=0$ and the external source is a point-source of the Dirac delta function, the magnitude of $\mathcal{Y}_{3, \infty} / Y_{\infty}^{2}$ is roughly expressed using factorial moments of $q$-neutrons (i.e., $\bar{q}, \overline{q(q-1)}$, and $\overline{q(q-1)(q-2)})$ because $Y_{\infty} \propto \overline{q(q-1)} / \bar{q}$ and $\mathcal{Y}_{3, \infty} \propto \overline{q(q-1)(q-2)} / \bar{q}$ :

$$
\frac{y_{3, \infty}}{Y_{\infty}^{2}} \approx \frac{\mathcal{S}_{1} \mathcal{S}_{3}}{\mathcal{S}_{2} \mathcal{S}_{2}} \approx \frac{\bar{q} \times \overline{q(q-1)(q-2)}}{(\overline{q(q-1)})^{2}} \text {. }
$$

\section{ACTUAL EXPERIMENTS}

Zero-power reactor noise experiments were carried out for the third-order neutron correlation technique. As explained the details in previous studies $[1,6]$, the measured time-series data were analyzed using the moving block bootstrap method to estimate statistical errors of neutron-correlation factors $\left(Y\right.$ and $\left.\mathcal{Y}_{3}\right)$ and the ratio $y_{3} / Y^{2}$.

\subsection{Non-multiplication System Driven by ${ }^{252} \mathrm{Cf}$ source}

To validate the difference in $y_{3, \infty} / Y_{\infty}^{2}$ from " 3 " in a non-multiplication system driven by a ${ }^{252} \mathrm{Cf}$ neutron source, an inhouse neutron detector was used for the reactor noise measurement, as shown in Figure 1. This detector was originally developed as a compact and highly efficient neutron diffractometer [7]. The diffractometer consisted of 79 scintillation detector rings, which were shaped with a flexible and square tube of Transparent RUbber SheeT type Eu:LiCaAlF 6 (TRUST Eu:LiCAF [8]) scintillator ( $\square 4 \mathrm{~mm}$ square) and wrapped around a supporting structure. These ring detectors were arranged to cover a large solid angle with respect to the center of diffractometer (i.e., the sample setting position) and to avoid overlapping each other. Consequently, the whole detector shape became a hollow rugby-ball shape. Scintillation light from each TRUST Eu:LiCAF scintillator was read out by using wavelength-shifting fibers (WLSFs). Each end 
of the WLSFs was connected to a multianode photomultiplier tube. Each PMT pixel signal was individually processed and readout by the LiTA12 system [9] to obtain the list-mode data of neutron detection time. In this experiment, unfortunately, two detector rings were dead. $\mathrm{A}^{252} \mathrm{Cf}$ spontaneous fission source $(\sim 530 \mathrm{kBq})$ was located at the center position of this detector. To increase the neutron count rate up to $\sim 1100 \mathrm{cps}$, the inner $\mathrm{Cf}$ source and whole detector were surrounded by polyethylene blocks and reflectors, respectively. The total measurement time of reactor noise was $\sim 45.7$ hours.

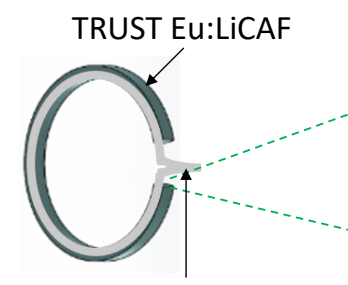

wavelengthshifting fiber

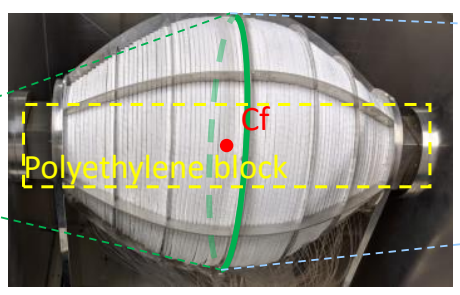

whole neutron detector

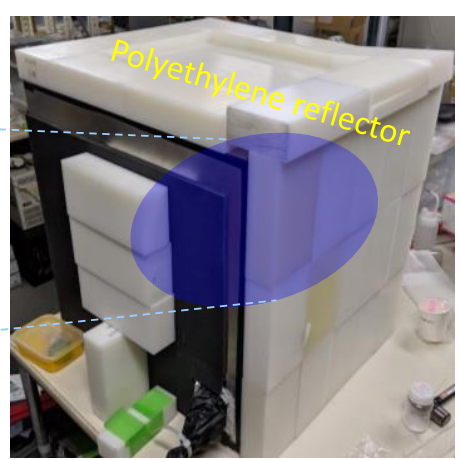

Figure 1. Overview of an experimental system to measure reactor noise in a non-multiplication system with ${ }^{252} \mathrm{Cf}$ spontaneous fission source.

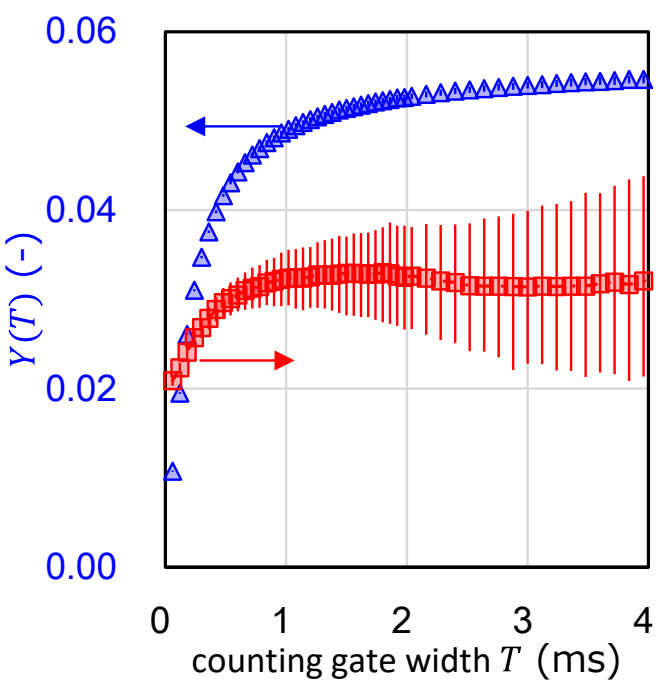

a) $Y(T)$ and $\mathcal{Y}_{3}(T)$
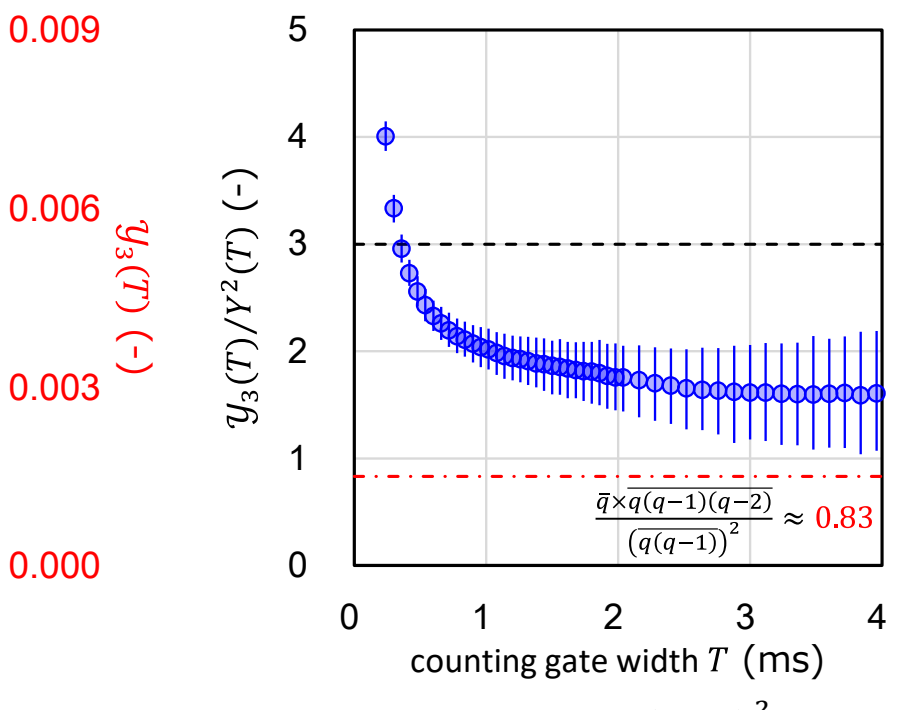

b) $\mathcal{Y}_{3}(T) /(Y(T))^{2}$

Figure 2. Experimental results of variations in neutron-correlation factors in a non-multiplication system with ${ }^{252} \mathrm{Cf}$ spontaneous fission source.

Figure 2 presents the variations in $Y(T), \mathcal{Y}_{3}(T)$, and $\mathcal{Y}_{3}(T) /(Y(T))^{2}$ with respect to the counting gate width $T$. In Figure 2, error bars indicate the bootstrap 95\% confidence intervals [1,6]. As a result, it was validated that the saturation value $\mathcal{Y}_{3, \infty} / Y_{\infty}^{2}$ was significantly different from the unique combination number " 3 ." In the case of this experiment, the saturation value $\mathcal{Y}_{3, \infty} / Y_{\infty}^{2}$ was roughly close to or larger than $\bar{q} \times \overline{q(q-1)(q-2)} /(\overline{q(q-1)})^{2} \approx 0.83$, which can be evaluated by the probability distribution 
$p_{\mathrm{S}}(q)$ of ${ }^{252} \mathrm{Cf}$ spontaneous fission [10]. Based on Fig. 2-a) and Eq. (18), the reasons for this discrepancy between measured $\mathcal{Y}_{3, \infty} / Y_{\infty}^{2}$ and 0.83 seems to be the (1) positive dead-time effect on $\mathcal{Y}_{3, \infty}$ and (2) neglect of spatial and neutron-energy effect on $\mathcal{Y}_{3, \infty} / Y_{\infty}^{2}$.

\subsection{Subcritical Systems Driven by Inherent Neutron Sources}

To investigate the difference in $\mathcal{Y}_{3, \infty} / Y_{\infty}^{2}$ from " 3 " in actual subcritical systems, reactor noise were passively measured at the following two Japanese experimental facilities under the shutdown state without any external neutron source such as ${ }^{252} \mathrm{Cf}$ or Am-Be neutron source: the Kindai University Training and Research Reactor (UTR-KINKI [11]), and the Kyoto University Critical Assembly (KUCA [1,6]).

The UTR-KINKI is a light-water-moderated and graphite-reflected two-core reactor. A fuel plate of the UTR-KINKI is highly-enriched uranium-aluminum alloy. Thus, there is a weak inherent neutron source, which is caused by $(\alpha, n)$ reactions of ${ }^{27} \mathrm{Al}$ due to the $\alpha$-decay of uranium isotopes. In the shutdown state, two safety rods and two shim rods were fully inserted. Then, the effective neutron multiplication factor $k_{\text {eff }}$ was relatively shallow, i.e. $k_{\text {eff }} \approx 0.98$. By setting a $\mathrm{BF}_{3}$ neutron detector at the center of the experimental core with the list-mode data acquisition system, zero-power reactor noise was measured for $\sim 15.8$ hours, where the neutron count rate was $\sim 39 \mathrm{cps}$.

As reported in previous studies [1,6], several series of reactor noise experiments were conducted in the Acore at the KUCA (A3/8"p36EU-NU), which is a polyethylene-moderated and -reflected core. The unit fuel cell consisted of one highly enriched highly-enriched uranium-aluminum alloy plate, one natural uranium plate, and three polyethylene plates. Thus, the core-average ${ }^{235} \mathrm{U}$ enrichment was $5.4 \mathrm{wt} \%$ and there was weak inherent neutron source mainly due to the spontaneous fission of ${ }^{238} \mathrm{U}$. In the shutdown state, three safety rods and three control rods were fully inserted, and moreover, $3 \times 3$ fuel and reflector assemblies were fully withdrawn. In addition, one normal and two partial fuel assemblies were replaced with the polyethylene reflector assemblies to achieve a deeper subcritical system $\left(k_{\mathrm{eff}} \approx 0.936\right)$, where the case name was denoted as "Shutdown+P" in the previous study [1]. In this experiment, four ${ }^{3} \mathrm{He}$ detectors were placed at the axial center positions of the ex-core reflector assemblies, where the total count rate was $\sim 21 \mathrm{cps}$. Using four detectors with the list-mode data acquisition system, zero-power reactor noise was measured for $\sim 15.6$ hours.

Figure 3 presents the variations in $Y(T), \mathcal{y}_{3}(T)$, and $\mathcal{y}_{3}(T) /(Y(T))^{2}$ with the bootstrap $95 \%$ confidence intervals for UTR-KINKI and KUCA, respectively. As can be seen from Fig. 3, the saturation values $y_{3, \infty} / Y_{\infty}^{2}$ were close to the unique combination number " 3 " at the critical state. Although the long-time measurements were carried out as much as possible, the statistical errors of $\mathcal{Y}_{3, \infty} / Y_{\infty}^{2}$ were large. Thus, in the case of the shallow subcritical system of UTR-KINKI, the differences from " 3 " were within the range of the $95 \%$ confidence intervals. On the other hand, in the case of the deep subcritical system of KUCA, the slight differences from " 3 " could be measured beyond the $95 \%$ confidence intervals. For example, when $T=0.02 \mathrm{~s},\left|\mathcal{Y}_{3, \infty} / Y_{\infty}^{2}-3\right| \approx 0.08$ with the $95 \%$ bootstrap confidence interval of $[0.02,0.15]$. This slight difference approximately corresponds to the magnitude of $-\rho \approx 0.07$. For further discussion, the reduction of the large statistical errors is necessary by increasing measurement time and improving the detection efficiency. In addition, instead of using the fundamental mode approximation, the investigation about the spatial and neutron energy effects on $\mathcal{Y}_{3, \infty} / Y_{\infty}^{2}$ is also desirable based on the numerical analysis based on the detector importance functions $[12,13]$. 


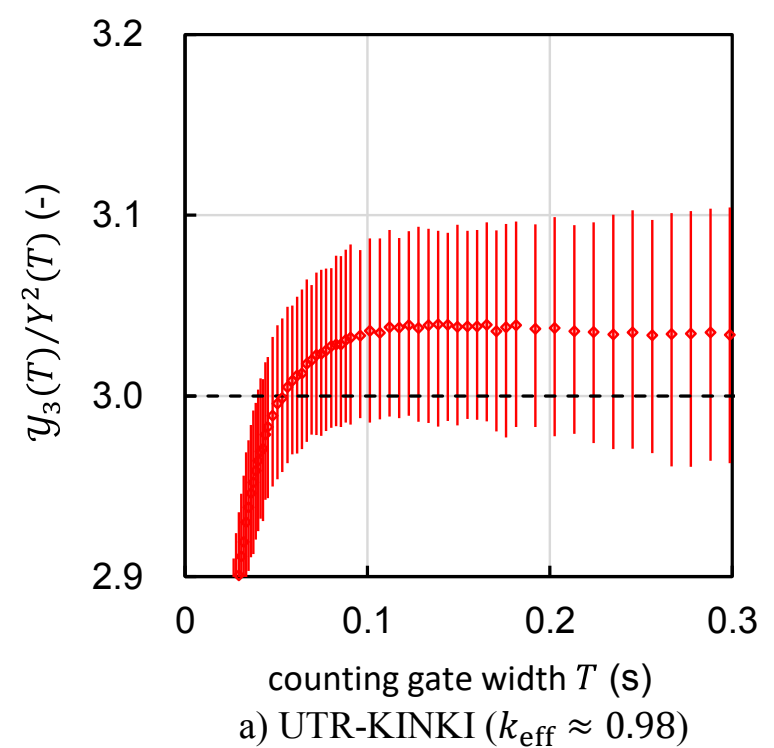

Figure 3. Experimental results of variations in $\mathcal{Y}_{3}(T) /(Y(T))^{2}$ in subcritical systems driven by inherent neutron sources.

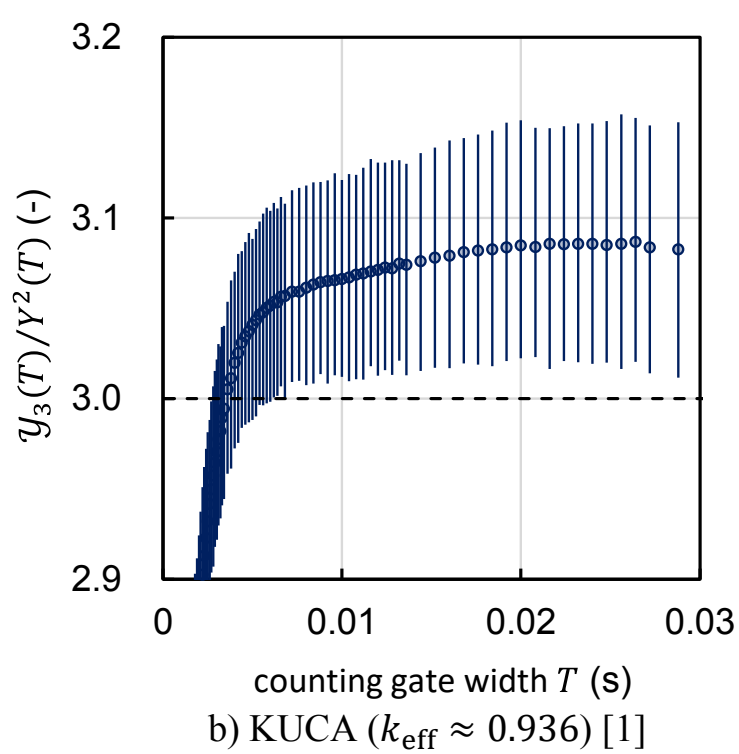

b) $\operatorname{KUCA}\left(k_{\mathrm{eff}} \approx 0.936\right)[1]$

\section{CONCLUSIONS}

In actual reactor noise analysis, the dead-time effect of a neutron detector could affect on the measurement results of the second- and third-order neutron-correlation factors $Y$ and $\mathcal{Y}_{3}$. Thus, the theoretical formulae for the third-order neutron correlation technique were newly derived based on the heuristic method with the fundamental mode approximation. Consequently, it was clarified that, $\mathcal{Y}_{3, \infty} / Y_{\infty}^{2}$ in the just critical state converges to the unique combination number " 3 " independent of the dead time. Through the actual reactor noise measurement carried out at the non-multiplication system driven by the ${ }^{252} \mathrm{Cf}$ source, it was demonstrated that the neutron-correlation ratio $\mathcal{Y}_{3, \infty} / Y_{\infty}^{2}$ was significantly different from the unique combination number " 3 " at the critical state. On the other hand, in the case of passive reactor noise measurement for a subcritical system under shutdown state, it was confirmed that $\mathcal{Y}_{3, \infty} / Y_{\infty}^{2}$ was close to " 3 " and the slight difference from " 3 " could be measured for the deep subcritical system. As presented in experimental results, the statistical errors of $\mathcal{Y}_{3, \infty} / Y_{\infty}^{2}$ tends to be large, thereby it is not easy to distinguish the statistically significant difference between $\mathcal{Y}_{3, \infty} / Y_{\infty}^{2}$ and " 3 ," as the subcriticality shallows. Thus, future research plans are the reduction of statistical errors and additional actual experiments for a deeper subcritical system.

\section{ACKNOWLEDGMENTS}

This work was carried out as the following two research programs: a joint research program of the UTRKINKI under the support of Graduate School of Engineering, Osaka University; and a visiting researcher's program of Kyoto University Institute for Integrated Radiation and Nuclear Science (KURNS). This work was supported by the Japan Society for the Promotion of Science (JSPS) Grant-in-Aid for Scientific Research (C) [Grant Number 19K05328]. 


\section{REFERENCES}

1. T. Endo, A. Yamamoto, M. Yamanaka, et al., "Experimental Validation of Unique Combination Numbers for Third- And Fourth-order Neutron Correlation Factors of Zero-power Reactor Noise," $J$. Nucl. Sci. Technol., 56(4), pp. 322-336 (2019).

2. T. Endo, Y. Yamane, and A. Yamamoto, "Space and Energy Dependent Theoretical Formula for the Third Order Neutron Correlation Technique," Ann. Nucl. Energy, 33(6), pp. 521-537 (2006).

3. Y. Yamane, and I. Pázsit, "Heuristic Derivation of Rossi-alpha Formula with Delayed Neutrons and correlated Source," Ann. Nucl. Energy, 25(17), pp. 1373-1382 (1998).

4. J.D. Orndoff, "Prompt Neutron Periods of Metal Critical Assemblies," Nucl. Sci. Eng., 2(4), pp. 450460 (1957).

5. Y. Yamane, and D. Ito, "Feynman- $\alpha$ Formula with Dead Time Effect for a Symmetric Coupled-core System," Ann. Nucl. Energy, 23(12), pp. 981-987 (1996).

6. T. Endo, and A. Yamamoto, "Comparison of Theoretical Formulae and Bootstrap Method for Statistical Error Estimation of Feynman- $\alpha$ Method," Ann. Nucl. Energy, 124, pp. 606-615 (2019).

7. S. Imai, K. Watanabe, A. Yamazaki, et al., "Response Evaluation of a Compact and Highly Efficient Neutron Diffractometer for Compact Accelerator Neutron Sources," Proceedings of ISORD-10, Taiyuan, China, July 16-19, 2019 (2019).

8. K. Watanabe, T. Yamazaki, D. Sugimoto, et al., "Wavelength-shifting Fiber Signal Readout from Transparent RUbber SheeT (TRUST) type LiCaAlF 6 Neutron Scintillator," Nucl. Instrum. Methods Phys. Res. A, 784, pp. 260-263 (2015).

9. S. Satoh, "Development of a New Exclusive Function for a 2012 Model ${ }^{6} \mathrm{Li}$ Time Analyzer Neutron Detector System", JPS Conf. Proc., 8, 051001 (2015).

10. R. Gwin, R.R. Spencer, and R.W. Ingle, "Measurements of the Energy Dependence of Prompt Neutron Emission from ${ }^{233} \mathrm{U},{ }^{235} \mathrm{U},{ }^{239} \mathrm{Pu}$, and ${ }^{241} \mathrm{Pu}$ for $\mathrm{E}_{\mathrm{n}}=0.005$ to $10 \mathrm{eV}$ Relative to Emission from Spontaneous Fission of ${ }^{252}$ Cf," Nucl. Sci. Eng., 87(4), pp. 381-404 (1984).

11. A. Sakon, K. Nakajima, S. Hohara, et al., "Experimental Study of Neutron Counting in a Zero-power Reactor Driven by a Neutron Source Inherent in Highly Enriched Uranium Fuels," J. Nucl. Sci. Technol., 56(2), pp. 254-259 (2019).

12. T. Endo, A. Yamamoto, Y. Yamane, "Development of Deterministic Code based on the Discrete Ordinates Method for the Third-order Neutron Correlation Technique," Ann. Nucl. Energy, 35(5), pp. 927-936 (2008).

13. P. Humbert, "Deterministic Transport Solution of Multiplicity Counting Equations," Proceedings of M\&C 2019, Portland, OR, August 25-29, 2019 (2019). 